\title{
Prediction of Left Main or 3-Vessel Disease Using Myocardial Perfusion Reserve on Dynamic Thallium-201 Single-Photon Emission Computed Tomography With a Semiconductor Gamma Camera
}

\author{
Shinya Shiraishi, MD, PhD; Fumi Sakamoto, MD, PhD; Noriko Tsuda, MD; \\ Morikatsu Yoshida, MD, PhD; Seiji Tomiguchi, MD, PhD; Daisuke Utsunomiya, MD, PhD; \\ Hisao Ogawa, MD, PhD; Yasuyuki Yamashita, MD, PhD
}

\begin{abstract}
Background: Myocardial perfusion imaging (MPI) may fail to detect balanced ischemia. We evaluated myocardial perfusion reserve (MPR) using TI dynamic single-photon emission computed tomography (SPECT) and a novel cadmium zinc telluride (CZT) camera for predicting 3-vessel or left main coronary artery disease (CAD).
\end{abstract}

Methods and Results: A total of 55 consecutive patients with suspected CAD underwent SPECT-MPI and coronary angiography. The MPR index was calculated using the standard 2-compartment kinetic model. We analyzed the utility of MPR index, other SPECT findings, and various clinical variables. On multivariate analysis, MPR index and history of previous myocardial infarction (MI) predicted left main and 3-vessel disease. The area under the receiver operating characteristic curve was 0.81 for MPR index, 0.699 for history of previous MI, and 0.86 for MPR index plus history of previous MI. MPR index $\leq 1.5$ yielded the highest diagnostic accuracy. Sensitivity, specificity, and accuracy were $86 \%, 78 \%$, and $80 \%$, respectively, for MPR index, $64 \%, 76 \%, 73 \%$ for previous $\mathrm{MI}$, and $57 \%, 93 \%$, and $84 \%$ for MPR index plus history of previous MI.

Conclusions: Quantification of MPR using dynamic SPECT and a novel CZT camera may identify balanced ischemia in patients with left main or 3-vessel disease. (Circ J 2015; 79: 623-631)

Key Words: Balanced ischemia; Myocardial perfusion reserve; Semiconductor gamma camera; Thallium-201; Three-vessel disease

M yocardial perfusion imaging (MPI) using singlephoton emission computed tomography (SPECT) is a widely used non-invasive imaging method for evaluation of ischemia and for risk stratification of patients with known or suspected coronary artery disease (CAD).,1,2 MPI plays a particularly important role in the identification of ischemic territories. Evaluation of myocardial perfusion defects on SPECT-MPI relies on the differential blood flow through the coronary arteries. Maximum-stress SPECT, however, may be normal in some patients with a global reduction in myocardial perfusion and show no focal perfusion defect despite the presence of left main or 3-vessel CAD. This results in the underestimation or misdiagnosis of ischemia in patients with balanced coronary ischemia.

\section{Editorial p 515}

Advances in nuclear cardiology due to the development of dedicated ultra-fast cardiac cameras able to provide high-quality, high-resolution, and high-diagnostic myocardial images s,4 $^{3,4}$ facilitate tomographic dynamic acquisition with the theoretical possibility of investigating radiotracer kinetics in vivo, and with 3-D positron emission tomography (PET)-like modalities.

The purpose of this study was to examine the diagnostic performance of the myocardial perfusion reserve (MPR) index calculated from dynamic ${ }^{201} \mathrm{Tl}$-chloride $(\mathrm{Cl})$ kinetic analysis using a cadmium zinc telluride (CZT) ultra-fast camera for predicting left main or 3-vessel disease on invasive coronary angiography obtained using conventional parameters.

Received August 21, 2014; revised manuscript received November 26, 2014; accepted November 28, 2014; released online January 14, 2015 Time for primary review: 18 days

Department of Diagnostic Radiology, Graduate School of Life Sciences (S.S., F.S., N.T., M.Y., D.U., Y.Y.), Department of Diagnostic Medical Imaging, School of Health Faculty of Life Sciences (S.T.), Department of Cardiovascular Medicine, Graduate School of Life Sciences (H.O.), Kumamoto University, Kumamoto, Japan

Mailing address: Shinya Shiraishi, MD, PhD, Department of Diagnostic Radiology, Graduate School of Life Sciences, Kumamoto University, 1-1-1 Honjyo, Chuo-ku, Kumamoto 860-8556, Japan. E-mail: shiraish@ kumamoto-u.ac.jp

ISSN-1346-9843 doi:10.1253/circj.CJ-14-0932

All rights are reserved to the Japanese Circulation Society. For permissions, please e-mail: cj@j-circ.or.jp 


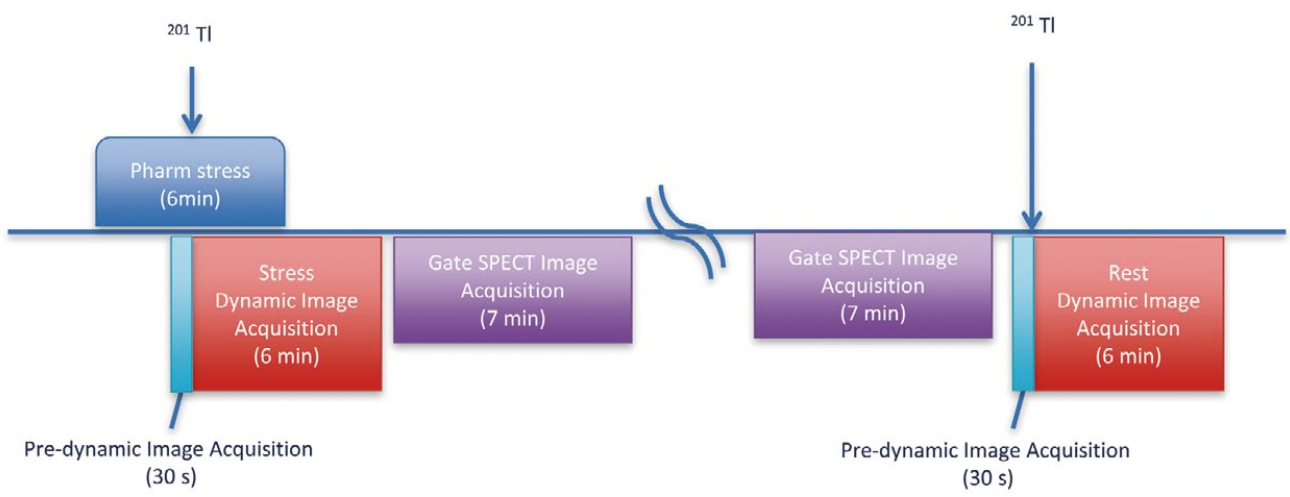

Figure 1. Single-photon emission computed tomography (SPECT) protocol. Adenosine is infused i.v. and symptoms, blood pressure, and electrocardiogram are monitored. A half-dose of $201 \mathrm{TI}$ is injected i.v. as a bolus $20 \mathrm{~s}$ after pre-dynamic image acquisition (30 s). Dynamic SPECT images are acquired for $6 \mathrm{~min}$ and conventional gated SPECT is performed for 7 min. At-rest images are acquired $3 \mathrm{~h}$ later, conventional gated SPECT is performed for $7 \mathrm{~min}$, a half-dose of ${ }^{201} \mathrm{Tl}$ is injected i.v. as a bolus $20 \mathrm{~s}$ after predynamic imaging (30s), and dynamic SPECT images are acquired for $6 \mathrm{~min}$.

\section{Methods}

\section{Subjects}

This study was approved by the institutional ethics board (no. 728) and all patients provided written prior informed consent.

We selected the subject group from a pool of 245 patients undergoing MPI-dynamic SPECT. We considered 55 consecutive patients ( 24 male, 31 female; mean age $74 \pm 10$ years) with suspected CAD eligible for this prospective study; they had undergone SPECT-MPI, and coronary angiography within 30 days after SPECT-MPI. Ineligible were patients who had undergone coronary artery bypass grafting or percutaneous coronary intervention before SPECT-MPI and patients with non-ischemic cardiomyopathy or severe valvular heart disease. Information on coronary risk factors was collected at the time of the imaging. Clinical risk factors were male gender, advanced age, body mass index, hypertension, diabetes mellitus, hyperlipidemia, chronic kidney disease (CKD), family history of CAD, previous myocardial infarction (MI), symptoms and ST segment depression on pharmacologic stress tests, at-rest and stress systolic blood pressure, Brinkman index, HbA1c, total-, low-density lipoprotein cholesterol, and high-density lipoprotein cholesterol, triglyceride, creatinine, estimated glomerular filtration rate, and brain natriuretic peptide.

\section{SPECT}

SPECT-MPI was performed using a CZT camera (Discovery NM530c; GE Healthcare) equipped with a multiple pinhole tungsten collimator and 19 stationary CZT detectors; 19 cardiac views are imaged simultaneously. Each detector yields high-quality 3-D images. Imaging was performed with the patient in the supine position with the arms placed over the head.

For stress MPI we induced pharmacologic vasodilator stress in all patients with i.v. adenosine $\left(0.120 \mathrm{mg} \cdot \mathrm{kg}^{-1} \cdot \mathrm{min}^{-1}\right.$ for $6 \mathrm{~min})$. We used $111 \mathrm{MBq}$ thallium $\left({ }^{201} \mathrm{Tl}\right)$ chloride injectable NMP (Nihon Medi-Physics) or a thallium chloride-201Tl injection (Fuji Film RI Pharma). At peak stress, we injected a halfamount of the radiotracer. Dynamic imaging was performed under a 6-min acquisition protocol in list mode; a bolus injection of the half-amount of the radiotracer was delivered simultaneously. A temporal series of 3-D axial volumes $(70 \times 70 \times 50$ voxels, $4 \times 4 \times 4 \mathrm{~mm}$ ) was reconstructed from the acquired raw data using maximum a posteriori-expectation maximization. This generated 72 3-D volumes integrating 5-s time frames in the course of $360 \mathrm{~s}$. Routine summed cardiac and gated stress images were acquired in list mode after dynamic imaging. Routine gated at-rest images were obtained $3 \mathrm{~h}$ after the administration of radiotracer.

For at-rest MPI we first performed a 30-s pre-scan to obtain a baseline image. The remaining half-amount of the radiotracer was injected in continuous fashion and sequential listmode dynamic scanning images were recorded in the same manner. We performed at-rest analysis by subtracting the baseline pre-scan image from the list-mode dynamic scans.

This was followed by summing cardiac and gated at-rest imaging results (Figure 1). The images were reformatted using Lister on a Xeleris workstation (GE Healthcare) and then reconstructed with a dedicated maximum penalized likelihood expectation maximization iterative algorithm (Myovation for Alcyon, GE Healthcare). Left ventricular function analysis was done using quantitative gated SPECT (Cedar-Sinai, Los Angeles, CA, USA). ${ }^{5}$ In patients with inadequate border detection we used manual editing.

Heart rate, blood pressure, and 12-lead electrocardiogram (ECG) were recorded at baseline and every $60 \mathrm{~s}$ during stress and at-rest imaging.

\section{Quantification of MPI}

List-mode dynamic acquisition images in DICOM format were transferred to a workstation for image analysis using inhouse software. It allows the automatic definition of a volume of interest (VOI) for the blood pool in the lumen of the left ventricle $(\mathrm{LV})$ and the whole $\mathrm{LV}$ wall on the global cardiac image (Figure 2). After the reconstruction process the drawn VOI is automatically shown on the reframed volume and aligned with the global volume. The VOI-related time-activity curve (TAC) can be extracted by averaging the signal intensity in the VOI in each time frame (Figure 2). The number of 

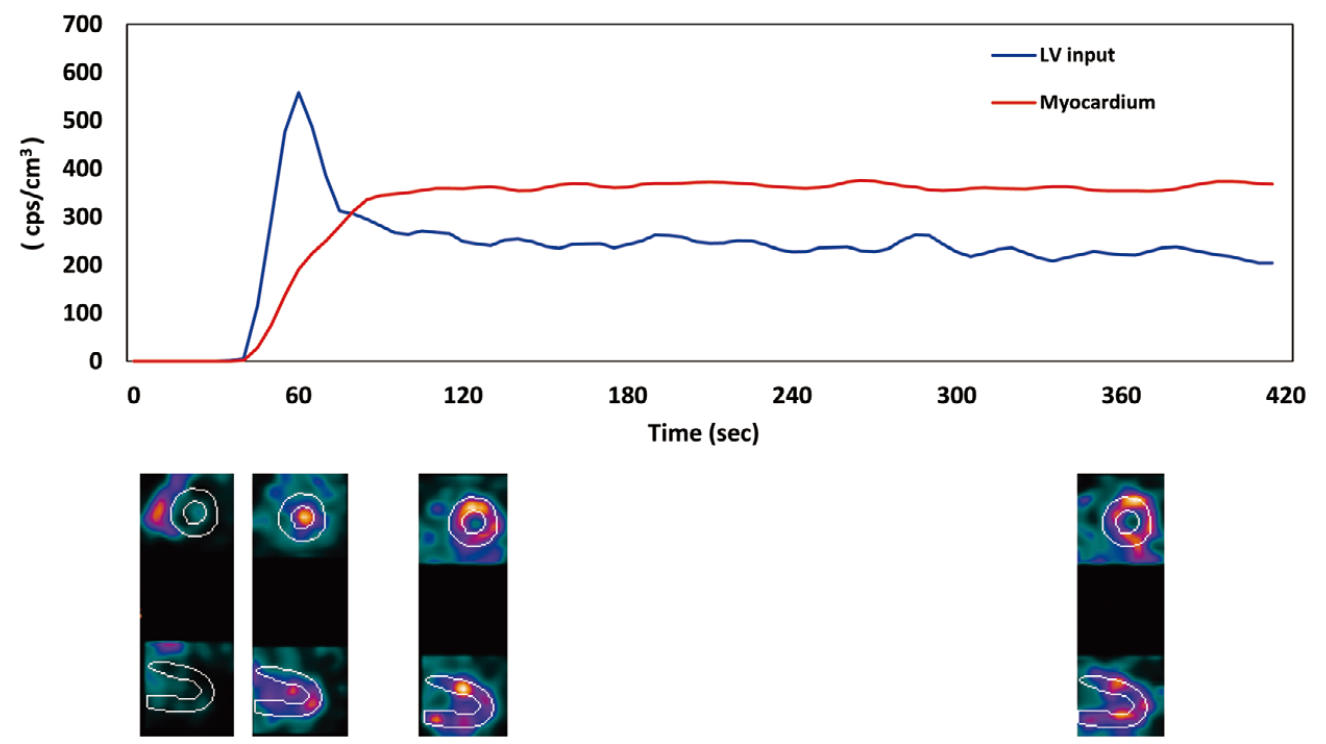

Figure 2. Dynamic acquisition results. Left ventricular (LV) blood pool and myocardial time-activity curves. Corresponding 5-s images, mid-ventricular short-axis, and vertical long-axis slice. LV input: VOI for blood pool in left ventricle lumen. Myocardium: VOI for whole left ventricle wall. VOI, volume of interest.

counts obtained was normalized to the VOI size $\left(\mathrm{mm}^{3}\right)$ and the frame duration, consequently, the TAC is expressed in counts per $\mathrm{mm}^{3} / \mathrm{s}$. Blood pool curves served as input functions in kinetic analysis. Global TAC were fitted to a 2-compartment kinetic model with input functions derived from factor analysis. $\mathrm{K}_{1}$ and $\mathrm{K}_{2}$ ( ${ }^{201} \mathrm{Tl}$ uptake) were calculated for the stress and at-rest images. MPR index was then calculated as the ratio of the stress and the at-rest $\mathrm{K}_{1}\left(\mathrm{MPR}\right.$ index $=\mathrm{K}_{1}$ stress $/ \mathrm{K}_{1}$ at-rest). Relative myocardial perfusion was also evaluated. For visual interpretation the images were blinded and re-oriented for short- and long-axis views. The evaluation was performed in terms of a representative system with the assistance of 2 experienced nuclear medicine physicians. We evaluated the images according to 17 -segment visual interpretation using a 5-point score. The summed stress, at-rest, and difference scores (SSS, SRS, SDS) were calculated. We derived the transient ischemic dilation (TID) ratio from non-gated acquisitions.

\section{Coronary Angiography}

All patients with no intervening coronary events, interventional procedures, or changes in symptoms underwent coronary angiography within 30 days after MPR-SPECT. Cineangiograms of the coronary arteries were obtained in multiple projections using a standard technique. The angiographic criterion used to define the presence of significant CAD was a visually determined stenosis diameter $\geq 50 \%$ in the left main artery and $\geq 75 \%$ in the left anterior descending, left circumflex, or right coronary artery and its main branch vessels. Vessels supplied by patent bypass grafts were considered patent. Significant stenosis was defined as coronary lesions with a stenosis diameter $\geq 75 \%$ or fractional flow reserve $<0.8$ in the left anterior descending, left circumflex, or right coronary artery and its main branch vessels, and stenosis diameter $\geq 50 \%$ in the left main trunk.

\section{Statistical Analysis}

We analyzed the utility of the MPR index, of other SPECT findings, and of various clinical variables for predicting left main or 3-vessel disease.

Continuous variables are expressed as mean (or median) and range or SD. Disease severity determined on angiography was assessed using 1-way ANOVA. Tukey-Kramer method was used for post-hoc tests of 1-way ANOVA. Multivariate forward stepwise regression analysis was used to evaluate the predictive ability for left main or 3-vessel disease. Receiver operating characteristic (ROC) curves were generated to evaluate the ability of selected continuous variables to predict left main or 3-vessel disease. Cut-offs were selected using ROC curves. Diagnostic accuracy was evaluated by calculating sensitivity, specificity, positive and negative predictive values (PPV, NPV), and accuracy. All statistical analysis was done using SPSS (version 21.0.0, SPSS).

\section{Results}

\section{Patient Characteristics}

We enrolled 55 patients, their mean age was $74 \pm 10$ years; 24 patients $(43.6 \%)$ were male. The patient characteristics and SPECT findings are given in Table 1.

Of the 55 patients, $45(81.8 \%)$ presented with obstructive CAD. This high incidence reflects the inclusion in the study of only patients with high pretest values in whom coronary angiography was required. Angiographically, 41 of the 55 patients $(74.5 \%)$ presented with no-vessel $(n=10,24.4 \%)$ or 1 -vessel $(n=18,43.9 \%)$ or 2 -vessel disease $(n=13,31.7 \%) ; 14$ of all patients $(25.5 \%)$ had left main or 3-vessel disease. One patient was diagnosed with left main, 10 with 3 -vessel disease, and 3 with left main plus 3-vessel disease.

Hypertension or CKD was present in $34(61.8 \%)$ and 29 patients $(52.7 \%)$, respectively, and 44 patients $(80 \%)$ took 


\begin{tabular}{|c|c|c|c|}
\hline Characteristic & $\begin{array}{c}\text { Angiographic } \\
0-2-\text { vessel CAD }(n=41)\end{array}$ & $\begin{array}{l}\text { Angiographic left main or } \\
\text { 3-vessel CAD }(n=14)\end{array}$ & P-value \\
\hline Male & $13(24)$ & $11(79)$ & 0.178 \\
\hline Hypertension & $22(73)$ & $12(86)$ & 0.503 \\
\hline Diabetes mellitus & $16(39)$ & $6(43)$ & 0.8 \\
\hline Hyperlipidemia & $20(49)$ & $9(64)$ & 0.316 \\
\hline CKD & $22(54)$ & $7(50)$ & 0.813 \\
\hline Familial history of CAD & $7(17)$ & $4(29)$ & 0.285 \\
\hline Previous MI & $10(23)$ & $9(64)$ & 0.009 \\
\hline Chest pain & $4(10)$ & $2(14)$ & 0.438 \\
\hline ST depression & $4(10)$ & $1(7)$ & 0.623 \\
\hline Age (years) & $73.5 \pm 11.1$ & $75.6 \pm 7.2$ & 0.519 \\
\hline BMI $\left(\mathrm{kg} / \mathrm{m}^{2}\right)$ & $24.2 \pm 5.0$ & $22.1 \pm 3.1$ & 0.151 \\
\hline Brinkman score & $431 \pm 538$ & $526 \pm 491$ & 0.563 \\
\hline HbA1c (\%) & $5.9 \pm 0.7$ & $5.9 \pm 0.9$ & 0.911 \\
\hline TC (mg/dl) & $157 \pm 34$ & $150 \pm 28$ & 0.495 \\
\hline HDL-C (mg/dl) & $48 \pm 13$ & $47 \pm 13$ & 0.736 \\
\hline LDL-C (mg/dl) & $89 \pm 29$ & $86 \pm 19$ & 0.684 \\
\hline $\mathrm{TG}(\mathrm{mg} / \mathrm{dl})$ & $115 \pm 70$ & $109 \pm 42$ & 0.736 \\
\hline $\mathrm{Cr}(\mathrm{mg} / \mathrm{dl})$ & $2.1 \pm 2.6$ & $1.8 \pm 2.2$ & 0.711 \\
\hline eGFR $\left(\mathrm{ml} \cdot \mathrm{min}^{-1} \cdot 1.73 \mathrm{~m}^{-2}\right)$ & $50 \pm 26$ & $55 \pm 25$ & 0.603 \\
\hline BNP (pg/ml) & $117 \pm 136$ & $552 \pm 1,557$ & 0.077 \\
\hline At-rest SBP & $135 \pm 26$ & $131 \pm 33$ & 0.603 \\
\hline Stress SBP & $119 \pm 25$ & $114 \pm 19$ & 0.515 \\
\hline At-rest LVEF & $59 \pm 15$ & $51 \pm 14$ & 0.073 \\
\hline Stress LVEF & $57 \pm 15$ & $48 \pm 15$ & 0.055 \\
\hline SSS & $7.1 \pm 6.6$ & $12.4 \pm 10.1$ & 0.085 \\
\hline SRS & $3.3 \pm 5.0$ & $7.2 \pm 7.3$ & 0.077 \\
\hline SDS & $3.9 \pm 3.6$ & $5.1 \pm 4.3$ & 0.293 \\
\hline TID & $1.08 \pm 0.14$ & $1.08 \pm 0.08$ & 0.903 \\
\hline $\mathrm{K}_{1}$ at rest & $0.020 \pm 0.007$ & $0.018 \pm 0.004$ & 0.301 \\
\hline $\mathrm{K}_{1}$ stress & $0.033 \pm 0.010$ & $0.025 \pm 0.010$ & 0.015 \\
\hline $\mathrm{K}_{2}$ at rest & $0.013 \pm 0.005$ & $0.013 \pm 0.004$ & 0.992 \\
\hline $\mathrm{K}_{2}$ stress & $0.019 \pm 0.007$ & $0.020 \pm 0.008$ & 0.838 \\
\hline MPR index & $1.71 \pm 0.39$ & $1.31 \pm 0.23$ & 0.001 \\
\hline
\end{tabular}

Data given as $\mathrm{n}(\%)$ or median \pm SD. BMI, body mass index; BNP, B-type natriuretic peptide; CAD, coronary artery disease; CKD, chronic kidney disease; eGFR, estimated glomerular filtration rate; HDL-C, high-density lipoprotein cholesterol; LDL-C, low-density lipoprotein cholesterol; LVEF, left ventricular ejection fraction; MI, myocardial infarction; MPR, myocardial perfusion reserve; SBP, systolic blood pressure; SDS, summed difference score; SPECT, single-photon emission computed tomography; SRS, summed stress score; SSS, summed stress score; TC, total cholesterol; TG, triglyceride; TID, transient ischemic dilation.

\begin{tabular}{|ccc|}
\hline \multirow{2}{*}{ Table 2. Multivariate Predictors of LMD/3-VD } \\
\cline { 2 - 3 } Variables & \multicolumn{2}{c|}{ Multivariate analysis } \\
\cline { 2 - 3 } Previous MI & OR $\mathbf{9 5 \%} \mathbf{~ C l})$ & P-value \\
MPR index & $7.54(1.50-37.99)$ & 0.014 \\
& $82.30(3.77-1,798.33)$ & 0.005 \\
\hline
\end{tabular}

$\mathrm{LMD}$, left main disease; VD, vessel disease. Other abbreviations as in Table 1.

medication to control their blood pressure and cholesterol.

\section{Severity of and Risk Factors for CAD}

The results of univariate analysis are shown in Table 1. Previous MI $(\mathrm{P}<0.009)$, stress $\mathrm{K}_{1}(\mathrm{P}<0.015)$, and MPR index $(\mathrm{P}<0.001)$ were significant independent predictors of severity of CAD. Subsequent multivariate forward stepwise regression analysis indicated that MPR index and history of previous MI were independent predictors of left main and 3-vessel CAD (Table 2). The median MPR index of the study population was 1.60 (range, 0.76-2.65). In the absence of vessel disease it was 1.79 (range, 1.36-2.45); 1-vessel disease, 1.72 (range, 0.95-2.65); 2-vessel disease, 1.58 (range, 1.07-2.10); and 3 -vessel disease, 1.33 (range, 0.76-1.72; Figure 3). The average MPR index in patients with no-, 1-, or 2-vessel disease was $1.71 \pm 0.39$. In contrast, in patients with left main or 3-vessel disease it was $1.31 \pm 0.23$; it was 1.35 in 1 patient with only left main disease, 1.29 in 10 patients with 3-vessel disease, and 1.33 in 3 patients with both left main disease and 3-vessel disease.

Of the 55 patients, 19 (34.5\%) had suffered previous MI. Earlier infarcts had occurred in 4 of 10 patients with no-vessel disease, 1 of 18 (5.6\%) with 1-vessel disease, 5 of 13 (38.5\%) 
A

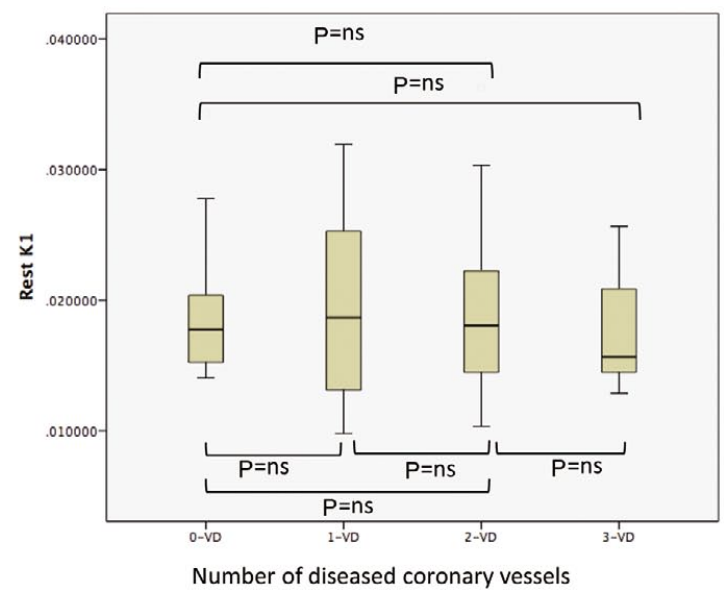

B

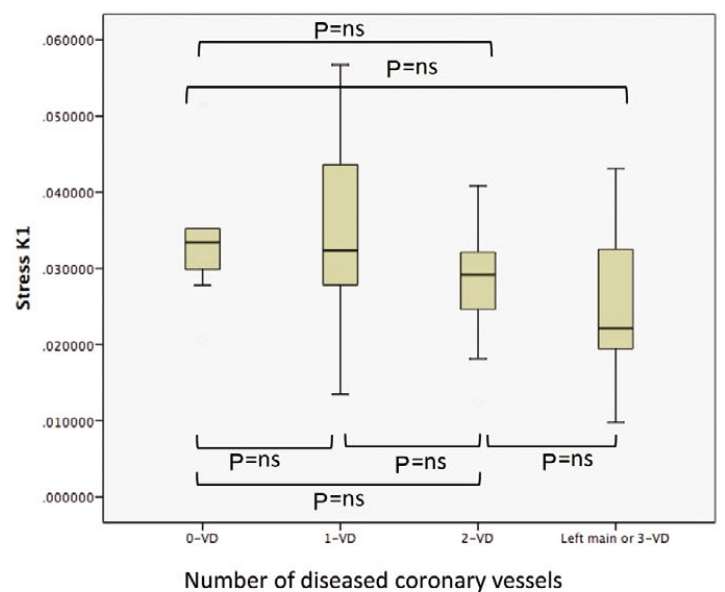

\section{C}

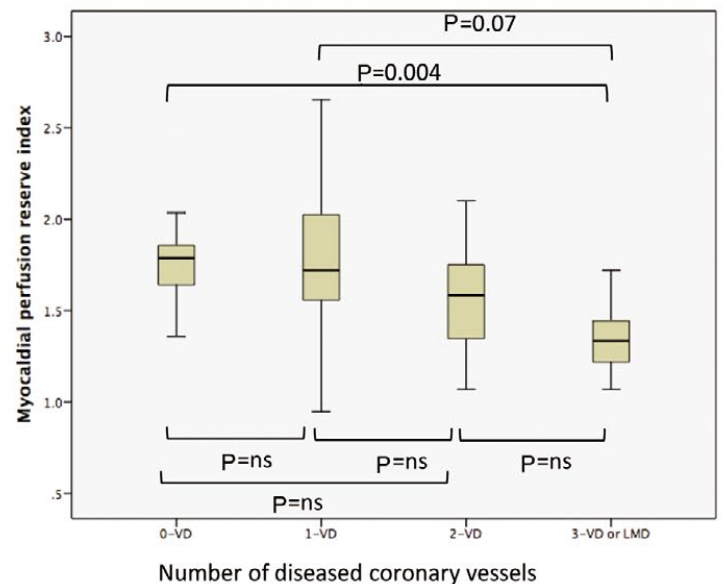

Figure 3. (A) Rest $K_{1}$, (B) stress $K_{1}$, and (C) myocardial perfusion reserve index vs. extent of angiographic disease. LMD, left main disease; $\mathrm{VD}$, vessel disease.

\begin{tabular}{|lccccc|}
\hline Table 3. Diagnostic Accuracy $(\mathbf{n}=\mathbf{5 5})$ & & & & & Accuracy \\
& Sensitivity & Specificity & PPV & NPV & Ac \\
MPR index & 86 & 78 & 57 & 94 & 80 \\
MPR index and previous MI & 50 & 95 & 78 & 85 & 69 \\
MPR index or previous MI & 100 & 59 & 45 & 100 & 73 \\
Previous MI & 64 & 76 & 47 & 86 & \\
\hline
\end{tabular}

MPR index cut-off, 1.5. NPV, negative predictive value; PPV, positive predictive value. Other abbreviations as in Table 1.

with 2-vessel disease, and 9 of 14 (64.3\%) patients with 3-vessel disease.

Area under the ROC curve for MPR index, for previous MI, and for a combination of these variables was $0.81,0.70$, and 0.84 , respectively. The MPR index cut-off $\leq 1.5$ yielded the best diagnostic accuracy. Sensitivity, specificity, and accuracy were $86 \%, 78 \%$, and $80 \%$ for MPR index; $64 \%, 76 \%$, and $73 \%$ for previous MI; and $50 \%, 95 \%$, and $84 \%$ for MPR index plus previous MI (Table 3 ).

\section{Discussion}

MPI plays an important role in the diagnosis and risk stratification of patients with symptoms of or documented CAD. ${ }^{6-8}$ Balanced 3-vessel CAD creates a diagnostic dilemma for the nuclear medicine physician. If the degree of disease is equivalent in all 3 main coronary arteries. As is the case in balanced 3 -vessel CAD, the radiotracer will show equal uptake in all portions of the myocardium and the results of myocardial perfusion scintigraphy may be false negative. In the Tanaka et al study, 4 of their 37 patients with intermediate-high 


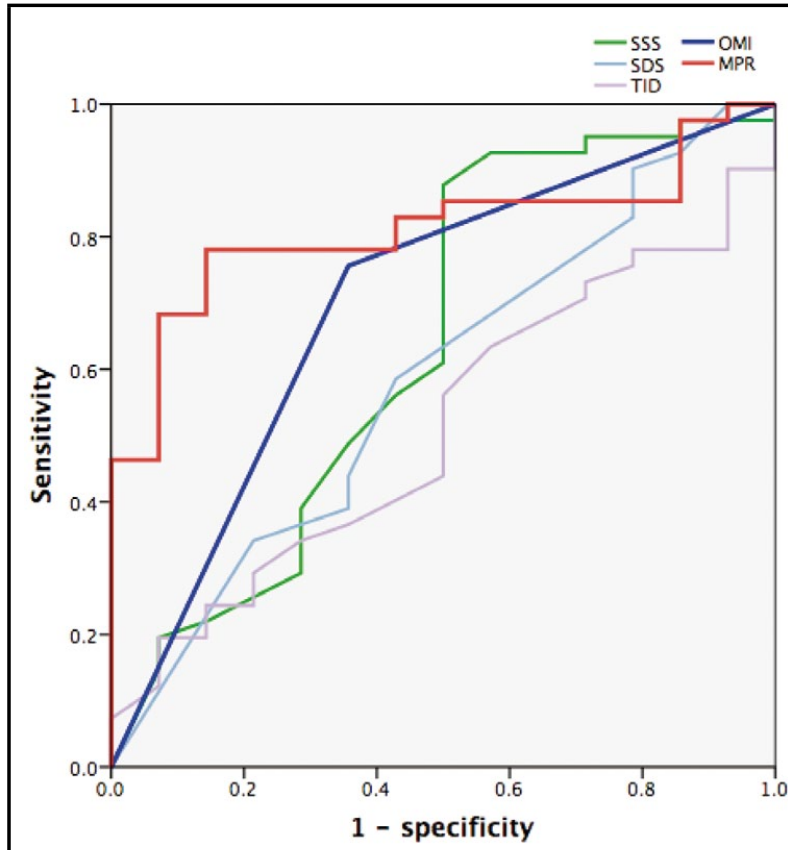

Figure 4. Receiver operator characteristic (ROC) analysis of the prediction of left main or 3-vessel coronary artery disease. The area under the curve is 0.81 for myocardial perfusion reserve (MPR), 0.70 for old myocardial infarction (OMI), 0.64 for summed stress score (SSS), and 0.51 for transient ischemic dilation (TID), respectively. SDS, summed difference score.

SYNTAX score, had \% ischemic difference score $<10 \%$; all 4 presented with left main or 3-vessel CAD. ${ }^{9}$ In those 4 patients the severity of myocardial ischemia may have been low. The major limitations of MPI are its spatial relativity in perfusion defect analysis. In addition, balanced myocardial ischemia may have been present in those patients with severe CAD, resulting in lower reversibility from stress to at rest. Although surrogate markers on SPECT-MPI, for example TID, ${ }^{10,11}$ increased post-stress end-systolic and/or end-diastolic volume, ${ }^{10}$ post-adenosine stress myocardial stunning, ${ }^{12}$ decrease in poststress LV ejection fraction, ${ }^{13}$ and increased post-stress tracer uptake in the right ventricle $(\mathrm{RV})^{14,15}$ may help to identify balanced ischemia, the utility of these markers is limited. TID and the increased post-stress end-systolic and/or end-diastolic volume are specific but not sensitive for severe and extensive CAD and generally signify worse prognosis. We postulate that the sensitivity of TID decreases more after adenosine- than exercise-load stress and that this explains why TID was not a significant predictive factor in the present study. The increased post-stress RV tracer uptake is sensitive but not specific for the detection of severe and extensive CAD. ${ }^{16}$ In exercise studies, ECG findings may be positive for ischemia; and in studies with adenosine (a strict vasodilator that rarely produces ischemia), the ischemic changes seen on ECG recordings may be due to the coronary artery steal phenomenon during an episode of maximum coronary artery vasodilation. Dilated cardiomyopathy and hypokinesis on gated images alert the nuclear medicine physician that the degree of CAD may be underestimated on SPECT.

Quantitative estimates of MPR that can be obtained with PET may be useful for the identification of balanced ischemia.
Ziadi et al concluded that the integration of quantitative flow analysis and standard relative ${ }^{82} \mathrm{Rb}$ PET-MPI could help to improve the detection of 3-vessel CAD. ${ }^{17}$ In $88 \%$ of their patients with severe 3-vessel CAD the myocardial flow reserve (MFR) was globally reduced $(<2) .{ }^{17}$ Fiechter et al evaluated the added diagnostic value of MFR on ${ }^{13} \mathrm{~N}$-ammonia PET/CT to predict angiographic CAD. ${ }^{18}$ Adding MFR to MPI reclassified one-third of all normal MPI findings and diagnostic accuracy increased from $79 \%$ to $92 \%(\mathrm{P}<0.005)$, primarily due to an increase in sensitivity from $79 \%$ to $96 \% .{ }^{18}$ Naya et al reported the largest angiographic study to date of the interaction between inducible myocardial ischemia, quantitative CFR, and extent and severity of CAD. ${ }^{19}$ They used ${ }^{82}$ Rb-PET to study 290 consecutive patients, 55 of whom presented with angiographic evidence of high-risk CAD involving left main disease and 2-vessel disease ( $>70 \%$ stenosis) or all 3 major epicardial coronary arteries. They found that normal MPR on MPI was associated with a low likelihood of high-risk CAD $(\mathrm{NPV}=97 \%) .{ }^{19}$

Earlier studies suggested the possibility of quantifying MPR on dynamic SPECT acquired with conventional SPECT parameters. ${ }^{20,21}$ Although these studies provided initial proof of concept, they also highlighted the limitations of conventional SPECT systems for the dynamic acquisition of tomographic data necessary for the quantification of rapid changes in radiotracer concentration. In addition, during rapid image acquisition, mechanical and patient safety constraints can limit the detector orbit to circular paths at increased distances from the patient, this results in degraded spatial resolution. This problem is compounded by the relatively limited temporal resolution of conventional scintillation crystals for collecting an adequate number of counts during rapidly changing tracer concentration. Semiconductor gamma cameras facilitate tomographic dynamic acquisitions with the theoretical possibility of investigating radiotracer kinetics in vivo and with a 3-D PET-like modality. Ben-Haim et al showed that the MPR derived from dynamic SPECT acquired with ${ }^{99 \mathrm{~m}} \mathrm{Tc}$-sestamibi and a dedicated solid-state cardiac camera was lower in patients with perfusion defects and in regions supplied by obstructed coronary arteries. ${ }^{22}$ They found that regions of abnormal perfusion identified on semi-quantitative analysis exhibited appropriate reductions in the MPR compared with the MPR of the visually normal remote myocardium. ${ }^{22}$ In patients undergoing coronary angiography, MPR index was lower in territories supplied by coronary arteries with at least $50 \%$ stenosis than in patients with non-obstructive disease (1.11 vs. 1.30). Problems of $99 \mathrm{mTc}$ radiopharmaceuticals include a low firstpass extraction fraction and a small distribution volume. This results in the underestimation of MPR.

In routine clinical practice, MPI using ${ }^{201} \mathrm{Tl}$ is well established for detecting exercise-induced myocardial ischemia and/or for assessing myocardial viability in patients with CAD. Due to its high first-pass extraction fraction ${ }^{23}$ and large distribution volume, ${ }^{201} \mathrm{Tl}$ has been considered an ideal tracer for the quantification of absolute myocardial blood flow not only at rest but also under hyperemic conditions. Compared with ${ }^{99} \mathrm{~m} \mathrm{Tc}$-sestamibi, ${ }^{24}$ the ${ }^{201} \mathrm{Tl}$ agent yields better linearity and a higher uptake, that is, $1-2 \%$ at rest and 3-5\% under stress vs. $1.0 \pm 0.4 \%$ at rest and $1.4 \pm 0.3 \%$ under stress. Therefore, to obtain the diagnosis of multi-vessel CAD we chose ${ }^{201} \mathrm{Tl}$ for the quantitative assessment of MBF on dynamic SPECT acquired with a dedicated ultra-fast cardiac camera. The radiation exposure is greater, however, with ${ }^{201} \mathrm{Tl}$ than $99 \mathrm{mTc}$ perfusion tracers; the reported average radiation exposure per study reported by the International Commission on 

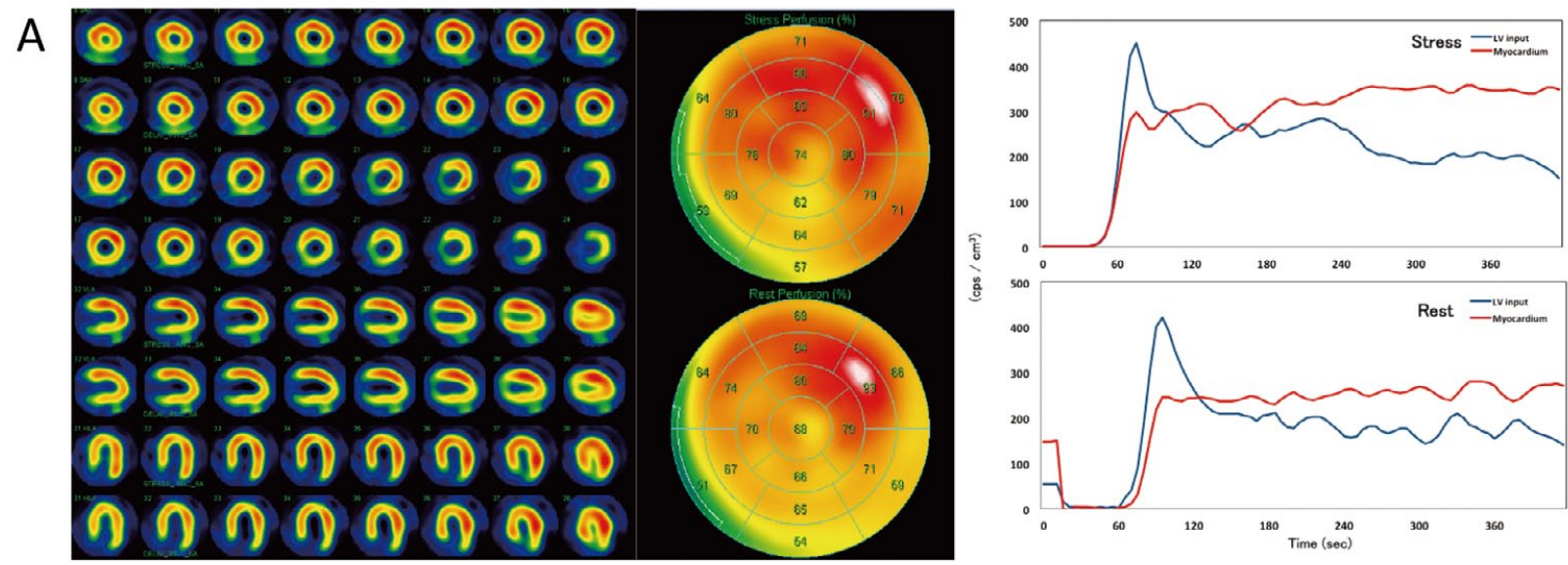

B
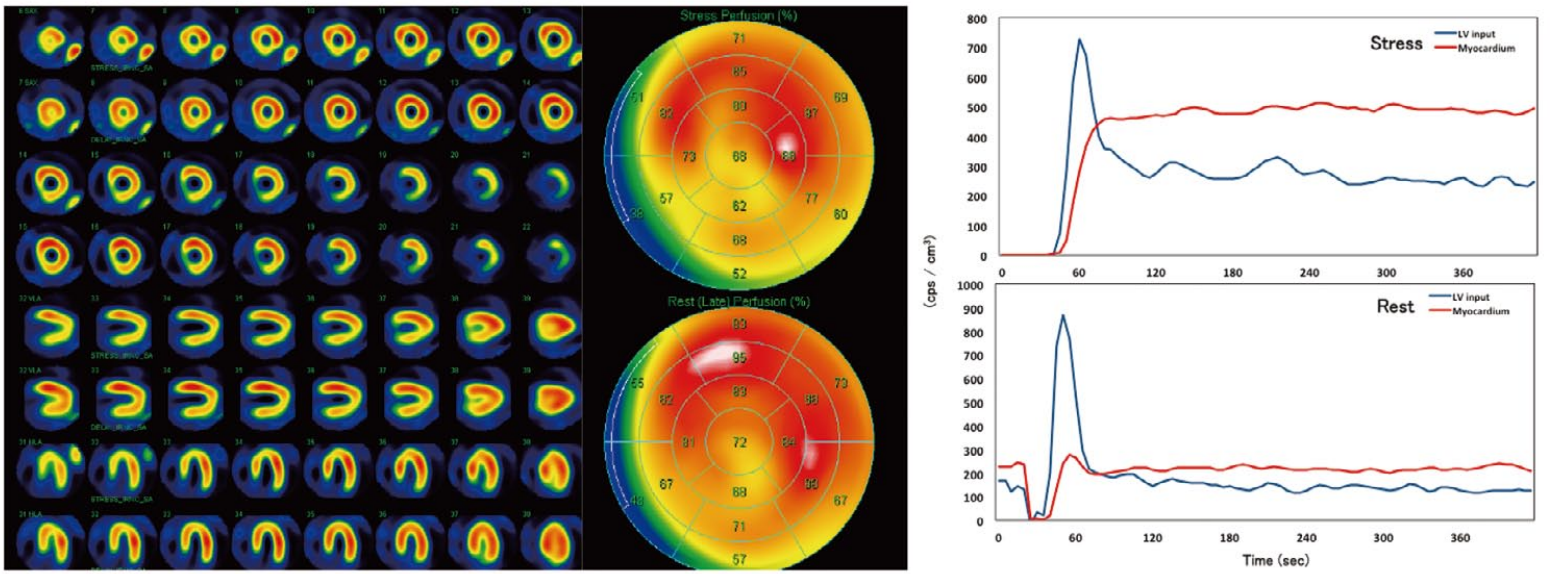

Figure 5. (A) Imaging for a 74-year-old man with 3-vessel stenosis on coronary angiography (segment 3, 25\%; segment 4 PD, 90\%; segment 7, 25\%; segment 8, 90\%; segment 9, 75\%; segment 11, 75\%; high lateral, 75\%). Myocardial perfusion reserve (MPR) index was 1.08. (B) A 71-year-old woman with no vessel stenosis on coronary angiography. MPR index was 2.45 . Attenuation in the inferior wall on both stress- and at-rest imaging in both patients suggests slightly low tracer accumulation. We judged this to reflect no redistribution in both cases. LV, left ventricle.

Radiological Protection was $15.5 \mathrm{mSv} .{ }^{25}$ In that study ${ }^{201} \mathrm{Tl}$ was injected twice for at-rest and stress scans, while in the present study we divided the injection solution in half for each scan to avoid increasing the radiation exposure dose. Even when the tracer dose is low, the high sensitivity and high resolution of the CZT camera yields high-quality images.

To our knowledge, this is the first evaluation of the feasibility of dynamic tomographic imaging and the quantification of myocardial perfusion and MPR for predicting severe human CAD on angiography acquired with a novel CZT camera system and ${ }^{201} \mathrm{Tl}-\mathrm{Cl}$. We found that previous $\mathrm{MI}$, stress $\mathrm{K}_{1}$, and MPR index were significant independent predictors of the extent of CAD. Subsequent multivariate forward stepwise regression analysis indicated that MPR index and history of previous MI were independent predictors of left main or 3-vessel CAD. We examined the diagnostic value of MPR and previous MI and of conventional indices such as SSS, SDS, and TID on ROC analysis (Figure 4). We found that ${ }^{201} \mathrm{Tl}$ MFR is a strong and better predictor than the standard relative MPI assessed using SSS or TID. We also showed that MFR quantified with ${ }^{201} \mathrm{Tl}$ MFR is an effective adjunct for the detection of severe $\mathrm{CAD}$ and that it may be of synergistic value beyond the relative MPI.

Although the mean of the at-rest $\mathrm{K}_{1}$ in patients with severe CAD was slightly low, it was relatively preserved in the presence of no- or up to 2-vessel CAD. A reduction in stress $\mathrm{K}_{1}$ and MPR index was correlated with the extent of CAD on angiography. This observation gives rise to the hypothesis that even if CAD progresses, myocardial perfusion is preserved to some degree by the autoregulation of coronary flow. In other words, quantification of at-rest myocardial perfusion is not useful for predicting CAD. Quantitative analysis of myocardial perfusion at steady-state maximum hyperemia and of MPR are more useful for predicting the extent of CAD. In Figure 5 we present 2 symmetrical cases. We also show that the combination of both variables was more accurate for predicting the extent of $\mathrm{CAD}$ than was each variable alone. We demonstrate that a history of previous MI was a useful predictor of the extent of CAD and that an increase in the number of previous MI events was correlated with the extent of CAD. It is necessary to note that the incidence of MI was similar in patients with no- or 2-vessel CAD.

We posit that the composition and vulnerability of plaque rather than plaque volume or the consequent severity of steno- 
sis are the most important determinants for the development of thrombus-mediated acute coronary syndrome. Compared to collagen-rich and hard plaque, lipid-rich and soft plaque are more unstable and rupture prone, and after their rupture they are highly thrombogenic. ${ }^{26}$ As shown in Table 3, when evaluated using MPR index alone, the sensitivity, specificity, PPV, and NPV for a diagnosis of severe CAD was $86 \%, 78 \%, 57 \%$, and $94 \%$, respectively. In addition, when both previous MI and MPR index were positive, the diagnostic value was more specific (specificity, 95\%; PPV, 78\%). When either previous MI or MPR index was positive, sensitivity was $100 \%$ (PPV, $45 \%$ ).

Based on the present findings we conclude that, in combination, MPR index and history of previous MI constitute a simple and reliable method for predicting severe CAD. The PPV of MFR with or without a history of previous MI, however, was modest. This suggests that quantitative flow estimates are a sensitive but potentially non-specific marker for obstructive epicardial CAD because a number of factors (eg, coronary risk factors, diffuse CAD, microvascular dysfunction) may affect MFR measurement and comorbidities may impair vascular reactivity. ${ }^{27}$

\section{Study Limitations}

The present study has some limitations. First, given that no gold standard such as PET was available to confirm the accuracy of the MPR index measurements, we carefully interpreted coronary angiographs. Second, in patients receiving pharmacotherapy the results of ${ }^{201} \mathrm{Tl}-\mathrm{Cl}$ MPI may fluctuate. No pre- and post-treatment ${ }^{201} \mathrm{Tl}-\mathrm{Cl}$ MPI studies were available with which to evaluate the potential effects of such treatment. Third, because corrections for attenuation and scatter were unavailable, quantification of the myocardial blood flow in terms of $\mathrm{ml} \cdot \mathrm{min}^{-1} \cdot \mathrm{g}^{-1}$ myocardium could not be obtained. Fourth, although regional flow measurements have been demonstrated to be of diagnostic value and can be linked to specific coronary stenoses, ${ }^{28,29}$ they may vary more than relative MPI data. ${ }^{7}$ The global MFR is of prognostic value ${ }^{9}$ but regional flow is not. We did not evaluate regional MPR in 17-segment- or per-vessel models because, for the calculation of MPR in such models, the detected counts were not sufficiently high for drawing a TAC, and TAC based on insufficient count increases the risk for measurement errors. Regional MPR analysis poses another risk and the evaluation of $\mathrm{K}_{1}$ for each region is difficult because attenuation or spill-over differ in different regions. Therefore we chose to use the whole LV rather than the regional MPR for evaluation. We suspect that defining MRP index as the ratio of the tracer uptake at stress and at rest offsets the attenuation effect on MPR, because attenuation was similar on both scans. Fifth, because heart rate and blood pressure at rest and under adenosine stress are different, myocardial blood flow is probably affected. We did not, however, correct $\mathrm{K}_{1}$ using the double product. Efforts are underway in our laboratory to develop software to address this issue. Last, the use of ${ }^{201} \mathrm{Tl}-\mathrm{Cl}$ SPECT raises concerns with respect to radiation exposure, and the low energy and long half-life of ${ }^{201} \mathrm{Tl}$ seriously limit its use in nuclear cardiology. Therefore we used lower doses of ${ }^{201} \mathrm{Tl}-\mathrm{Cl}$ for routine at-rest and stress tests. Additional prospective studies are under way in our laboratory.

\section{Conclusions}

Dynamic SPECT imaging and quantification of myocardial perfusion and MPR using a novel SPECT CZT camera system are useful for predicting left main and 3-vessel CAD. The present study must be viewed as a hypothesis-generating study and investigations using larger populations are required.

\section{Acknowledgments}

Noboru Katsuda, Ryuji Ikeda, Takayuki Kanenawa, and Eri Takenaga provided invaluable assistance in the performance of the SPECT. We thank Kazuo Hamada and Kazunori Kobayasi (Nihon Medi-Physics Japan) for supplying the dynamic analysis software.

\section{Disclosures}

Conflicts of Interest: None declared.

\section{Grants}

Grant-in-Aid for Scientific Research.

\section{References}

1. Hendel RC, Berman DS, Di Carli MF, Heidenreich PA, Henkin RE, Pellikka PA, et al. ACCF/ASNC/ACR/AHA/ASE/SCCT/SCMR/ SNM 2009 appropriate use criteria for cardiac radionuclide imaging: A report of the American College of Cardiology Foundation Appropriate Use Criteria Task Force, the American Society of Nuclear Cardiology, the American College of Radiology, the American Heart Association, the American Society of Echocardiography, the Society of Cardiovascular Computed Tomography, the Society for Cardiovascular Magnetic Resonance, and the Society of Nuclear Medicine. Circulation 2009; 119: e561-e587, doi:10.1161/CIRCULATIONAHA. 109.192519.

2. Shaw LJ, Hage FG, Berman DS, Hachamovitch R, Iskandrian A. Prognosis in the era of comparative effectiveness research: Where is nuclear cardiology now and where should it be? J Nucl Cardiol 2012; 19: 1026-1043.

3. Garcia EV, Faber TL, Esteves FP. Cardiac dedicated ultrafast SPECT cameras: New designs and clinical implications. J Nucl Med 2011; 52: 210-217.

4. Tanaka H, Chikamori T, Tanaka N, Hida S, Igarashi Y, Yamashita $\mathrm{J}$, et al. Diagnostic performance of a novel cadmium-zinc-telluride gamma camera system assessed using fractional flow reserve. Circ J 2014; 78: $2727-2734$.

5. Germano G, Erel J, Lewin H, Kavanagh PB, Berman DS. Automatic quantitation of regional myocardial wall motion and thickening from gated technetium-99m sestamibi myocardial perfusion single-photon emission computed tomography. J Am Coll Cardiol 1997; 30: $1360-1367$.

6. Berman DS, Kang X, Slomka PJ, Gerlach J, de Yang L, Hayes SW, et al. Underestimation of extent of ischemia by gated SPECT myocardial perfusion imaging in patients with left main coronary artery disease. J Nucl Cardiol 2007; 14: 521-528.

7. Parkash R, deKemp RA, Ruddy TD, Kitsikis A, Hart R, Beauchesne $\mathrm{L}$, et al. Potential utility of rubidium 82 PET quantification in patients with 3-vessel coronary artery disease. J Nucl Cardiol 2004; 11: 440-449.

8. Aziz EF, Javed F, Alviar CL, Herzog E. Triple vessel coronary artery disease presenting as a markedly positive stress electrocardiographic test and a negative SPECT-TL scintigram: A case of balanced ischemia. Heart Int 2011; 6: e22, doi:10.4081/hi.2011.e22.

9. Tanaka H, Chikamori T, Hida S, Igarashi Y, Shiba C, Usui Y, et al. Relationship of SYNTAX score to myocardial ischemia as assessed on myocardial perfusion imaging. Circ J 2013; 77: 2772-2777.

10. Heston TF, Sigg DM. Quantifying transient ischemic dilation using gated SPECT. J Nucl Med 2005; 46: 1990-1996.

11. Weiss AT, Berman DS, Lew AS, Nielsen J, Potkin B, Swan HJ, et al. Transient ischemic dilation of the left ventricle on stress thallium-201 scintigraphy: A marker of severe and extensive coronary artery disease. J Am Coll Cardiol 1987; 9: 752-759.

12. Hida S, Chikamori T, Tanaka H, Igarashi Y, Shiba C, Hatano T, et al. Postischemic myocardial stunning is superior to transient ischemic dilation for detecting multivessel coronary artery disease. Circ J 2012; 76: 430-438.

13. Johnson LL, Verdesca SA, Aude WY, Xavier RC, Nott LT, Campanella MW, et al. Postischemic stunning can affect left ventricular ejection fraction and regional wall motion on post-stress gated sestamibi tomograms. J Am Coll Cardiol 1997; 30: 1641 1648.

14. Williams KA, Schneider CM. Increased stress right ventricular activity on dual isotope perfusion SPECT: A sign of multivessel and/or 
left main coronary artery disease. J Am Coll Cardiol 1999; 34: 420-427.

15. Mannting F, Zabrodina YV, Dass C. Significance of increased right ventricular uptake on ${ }^{99 \mathrm{~m} T c-s e s t a m i b i}$ SPECT in patients with coronary artery disease. J Nucl Med 1999; 40: 889-894.

16. Higgins JP, Higgins JA, Williams G. Stress-induced abnormalities in myocardial perfusion imaging that are not related to perfusion but are of diagnostic and prognostic importance. Eur J Nucl Med Mol Imaging 2007; 34: 584-595.

17. Ziadi MC, Dekemp RA, Williams K, Guo A, Renaud JM, Chow BJ, et al. Does quantification of myocardial flow reserve using rubidium-82 positron emission tomography facilitate detection of multivessel coronary artery disease? J Nucl Cardiol 2012; 19: 670-680.

18. Fiechter M, Ghadri JR, Gebhard C, Fuchs TA, Pazhenkottil AP, Nkoulou RN, et al. Diagnostic value of $13 \mathrm{~N}$-ammonia myocardial perfusion PET: Added value of myocardial flow reserve. $\mathrm{J} \mathrm{Nucl} \mathrm{Med}$ 2012; 53: 1230-1234.

19. Naya M, Murthy VL, Taqueti VR, Foster CR, Klein J, Garber M, et al. Preserved coronary flow reserve effectively excludes high-risk coronary artery disease on angiography. J Nucl Med 2014; 55: $248-255$.

20. Ito Y, Katoh C, Noriyasu K, Kuge Y, Furuyama H, Morita K, et al. Estimation of myocardial blood flow and myocardial flow reserve by ${ }^{99 m} \mathrm{Tc}$-sestamibi imaging: Comparison with the results of $\left[{ }^{15} \mathrm{O}\right] \mathrm{H}_{2} \mathrm{O}$ PET. Eur J Nucl Med Mol Imaging 2003; 30: 281 - 287.

21. Tsukamoto T, Ito Y, Noriyasu K, Morita K, Katoh C, Okamoto H, et al. Quantitative assessment of regional myocardial flow reserve using Tc-99 m-sestamibi imaging. Circ J 2005; 69: 188-193.
22. Ben-Haim S, Murthy VL, Breault C, Allie R, Sitek A, Roth N, et al. Quantification of myocardial perfusion reserve using dynamic SPECT imaging in humans: A feasibility study. J Nucl Med 2013; 54: $873-879$.

23. Weich HF, Strauss HW, Pitt B. The extraction of thallium-201 by the myocardium. Circulation 1977; 56: 188-191.

24. Maddahi J. Properties of an ideal PET perfusion tracer: New PET tracer cases and data. J Nucl Cardiol 2012; 19(Suppl 1): S30-S37.

25. ICRP. Radiation dose to patients from radiopharmaceuticals: Addendum 3 to ICRP Publication 53: ICRP Publication 106: Approved by the Commission in October 2007. Ann ICRP 2008; 38: 1-197.

26. Falk E, Shah PK, Fuster V. Coronary plaque disruption. Circulation 1995; 92: 657-671.

27. Naya M, Murthy VL, Blankstein R, Sitek A, Hainer J, Foster C, et al. Quantitative relationship between the extent and morphology of coronary atherosclerotic plaque and downstream myocardial perfusion. J Am Coll Cardiol 2011; 58: 1807-1816.

28. Kajander S, Joutsiniemi E, Saraste M, Pietila M, Ukkonen H, Saraste A, et al. Cardiac positron emission tomography/computed tomography imaging accurately detects anatomically and functionally significant coronary artery disease. Circulation 2010; 122: 603-613.

29. Hajjiri MM, Leavitt MB, Zheng H, Spooner AE, Fischman AJ, Gewirtz H. Comparison of positron emission tomography measurement of adenosine-stimulated absolute myocardial blood flow versus relative myocardial tracer content for physiological assessment of coronary artery stenosis severity and location. JACC Cardiovasc Imaging 2009; 2: 751-758. 